\title{
DOSSIER
}

RAPHAËL SUIRE

CREM-CNRS, université de Rennes 1 JÉRÔME VICENTE

LEREPS, université de Toulouse

\section{Théorie économique des clusters et management des réseaux d’entreprises innovantes}

L'article propose une analyse de la performance collective des pôles de compétitivité basée sur les liens qui peuvent être établis entre l'économie de la connaissance et la théorie des clusters. Si les clusters tirent leur attractivité de leur charisme géographique et des flux de connaissances qui y circulent, les auteurs montrent que la proximité géographique entre les firmes innovantes est loin d'être une condition de leur performance et de leur pérennité. Parce que la connaissance est un bien systémique et complexe, les firmes doivent mixer des stratégies d'appropriation et d'accessibilité aux connaissances selon les phases de la chaîne de valeur de la connaissance. Cette organisation collective retira un avantage de la proximité géographique dans certaines conditions. 
I nitiée en 2005, la politique des pôles de compétitivité s'inscrit dans le cadre d'un programme plus global qui vise à répondre aux faiblesses de la France dans l'économie de la connaissance, conformément aux objectifs de Lisbonne qui visent à accroître le potentiel européen d'innovation. Il serait hasardeux à ce stade de proposer une évaluation précise des résultats de cette politique. Les outputs qui peuvent être le plus directement attendus (meilleure position de la France dans la hiérarchie européenne des déposants de brevets, des publications scientifiques) ne peuvent se faire sentir dans une période aussi courte alors même que l'implémentation de cette politique est toujours en cours, et que d'autres politiques complémentaires favorisant la recherche et l'innovation viennent encore s'y adjoindre. Il est néanmoins possible de proposer un cadre permettant de capturer les principaux déterminants de la réussite d'une telle politique. Le cadre proposé ici part des grands principes de l'économie de la connaissance et de la théorie économique des clusters. L'économie de la connaissance est une discipline qui a considérablement évolué ces dix dernières années, en particulier sur l'importance de la structuration des collectifs (dans) et des formes d'incitation (à) la production de connaissances (Antonelli, 2006). Ces grands principes seront mobilisés pour élaborer une analyse de la performance des clusters. Partant des formes d'externalités qui gouvernent la dynamique de colocalisation (Vicente, Suire, 2007; Suire, Vicente, 2008), il est possible de montrer l'ambivalence que génèrent les externalités locales de connaissances sur la dynamique de production de connaissances à l'œuvre dans les clusters. En effet, par-delà le charisme géo- graphique des clusters (Appold, 2005), c'est dans l'organisation des réseaux que réside leur performance, la proximité géographique entre les firmes innovantes étant alors bien loin d'être une garantie de la production de connaissances.

Formellement, la première partie fait le lien entre économie de la connaissance et théorie des clusters afin de dégager des hypothèses sur les propriétés de stabilité et d'attractivité des clusters. L'idée générale consiste à montrer que la connaissance est un bien systémique et complexe qui nécessite de la part des firmes de combiner des stratégies d'appropriation (des) et d'accessibilité (aux) connaissances. Ainsi dans la chaîne de valeur de la connaissance, la colocalisation des firmes peut être une stratégie rationnelle visant à favoriser l'accessibilité alors qu'elle peut s'avérer néfaste dès lors que la proximité géographique engendre des risques de sous-appropriation. Partant du constat que l'approche par les firmes dans le cadre de l'économie de la connaissance ne suffit pas, la deuxième partie se réapproprie les déterminants de la production de connaissances, en insistant sur une approche socio-économique des formes de réseaux sociaux qui favorisent l'attractivité des clusters et la performance des pôles de compétitivité.

\section{I. ÉCONOMIE DE LA CONNAISSANCE ET THÉORIE DES CLUSTERS}

Dans les «principes d'économie politique » (1870) Alfred Marshall pose les fondements de la théorie moderne des clusters. Bien avant de parler d'économies d'agglomérations et d'effets externes, Marshall pensait simplement que ces collectifs 
industriels relevaient du bon sens économique. En effet, en se rapprochant les unes des autres, " les secrets » des entreprises cessent pour ainsi dire d'être des secrets. D'une certaine manière, ils deviennent des composants élémentaires de l'atmosphère du territoire ou du district.

« On sait apprécier le travail bien fait, on discute aussitôt les mérites des inventions et des améliorations qui sont apportées aux machines, aux procédés et à l'organisation générale de l'industrie. Si quelqu'un trouve une idée nouvelle, elle est aussitôt reprise par d'autres, et combinée avec des idées nouvelles. Bientôt des industries subsidiaires naissent dans le voisinage, fournissant à l'industrie principale les instruments et les matières premières, organisant son trafic, et lui permettant de faire bien des économies diverses » (Livre IV, Ch X).

Est-ce cependant aussi simple que le décrit Marshall ou pour le dire autrement est-ce que la proximité géographique se suffit à elle-même pour retirer un bénéfice de l'appartenance au collectif, tel un pôle de compétitivité? C'est bien là un enjeu de taille pour juger de la performance des territoires innovants. À l'heure où la politique de « clusterisation » semble dominer l'action publique, le risque existe de se satisfaire d'un simple regroupement de façade ou d'un simple affichage d'une juxtaposition d'activités et d'agents.

Les liens entre économie de la connaissance et théories des clusters sont au cœur de nombreux travaux de recherche depuis les années 1990 (Cooke, 2002; Boschma, 2005; Iammarino et Mc Cann, 2006). Les clusters seraient des lieux avec un fort indice d'innovation collective en raison d'une meilleure exploitation des externalités de connaissances dont chacune des firmes pourrait profiter du fait de leur localisation à proximité d'autres firmes innovantes. L'idée de base qui soutient cet argument est que la connaissance est un bien public impur (Arrow, 1962), du fait de son caractère tout ou partiellement tacite (Foray, 2004), dont la diffusion s'accommode mieux d'une proximité géographique. L'idée est séduisante mais n'est pas exempte de limites. D'une part, empiriquement, certains clusters exercent un fort pouvoir d'attractivité alors que d'autres déclinent (Dalla Pria et Vicente, 2006; Suire et Vicente, 2008), et certains clusters présentent une forte instabilité (Longhi, 1999), voire des cycles de vie (Ter Wal et Boschma, 2007). D'autre part, d'un point de vue théorique, la définition même des externalités de connaissance s'apparente le plus souvent à une boîte noire (Breschi et Lissoni, 2001), si bien qu'il est difficile de capturer ce qui relève de la géographie de ce qui relève d'autres modes de diffusion des connaissances au sein des clusters.

Nous proposons de dépasser cette limite en nous appuyant sur d'autres propriétés de la connaissance, son caractère complexe, systémique et collectif (Antonelli, 2006 ; Sorenson et al., 2006). Ces propriétés permettront de comprendre ce qui, au-delà du charisme géographique des clusters (Appold, 2005), constitue les ressorts de leur performance.

\section{Le dilemme entre appropriation et accessibilité à la connaissance}

En tant qu'output, la connaissance, dans la tradition de Arrow (1962), possède les caractéristiques d'un bien public, aussi impur soit-il du fait des problèmes de codification et de transmission qu'elles engendrent (tableau 1). Dans ce cadre-là, les 
firmes doivent alors adopter des stratégies d'innovation basées sur l'exercice du droit de la propriété intellectuelle et sur une structure de gouvernance favorisant l'intégration de toutes les étapes de la chaîne de valeur de la connaissance. Ainsi elles limitent les risques de sous-appropriation des bénéfices de leur activité innovante et maintiennent leur incitation à innover par la possibilité que leur confère le droit de la propriété intellectuelle d'exercer un pouvoir de monopole.

Mais au-delà de cette propriété de bien public, la connaissance possède d'autres caractéristiques qui remettent quelque peu en cause ce modèle traditionnel. La connaissance est un bien complexe, systémique et, in fine, collectif. En effet, la connaissance est également un input qui entre dans la fonction de production des firmes. Pour produire de la connaissance, il faut recombiner et articuler des connaissances existantes (Sorenson et al., 2006). Cette propriété dégage au moins une implication majeure: les firmes privilégiant des formes de gouvernance basées exclusivement sur l'appropriation des bénéfices de l'innovation et l'intégration de la chaîne de valeur de la connaissance peuvent faire face à un déficit d'accessibilité à la connaissance qui provient de l'extérieur. Dans un contexte macro-économique où le progrès technologique provient essentiellement de la convergence technologique et sectorielle (TIC, biotechnologies, nanotechnologies, systèmes embarqués, technologies de mobilité, de sécurité, etc.), ce dilemme entre appropriation (des) et accessibilité (aux) connaissances devient de plus en plus saillant et élargit les formes de gouvernance possibles des activités innovantes.
La nécessité pour les firmes d'articuler des connaissances internes et externes les conduisent à combiner des stratégies d'appropriation et d'accessibilité à travers des stratégies adaptées de réseaux et d'alliances (Mowery et al., 1996; Hagerdoorn et al., 2000; Brossard et Vicente, 2007). D'un côté, elles doivent contrôler les externalités de leur activité de production de connaissances afin de tirer les bénéfices de leur exploitation sur les marchés. Pour cela, elles doivent extraire une rente de cette activité en fixant un prix pour l'usage de celleci (le droit de la propriété intellectuelle internalise l'externalité en réintroduisant la connaissance sur le marché via l'exercice d'un droit de propriété). De l'autre côté, elles doivent maintenir des conditions appropriées d'accessibilité à des connaissances externes complémentaires afin d'explorer de nouvelles opportunités technologiques et examiner la viabilité de nouveaux processus de recombinaison. Des stratégies de partenariat sont alors mobilisées par les firmes, lorsque ces dernières perçoivent un avantage à ouvrir mutuellement et selon divers degrés leur base de connaissance au partenaire.

Suivant Antonelli (2006), la connaissance comme bien complexe et systémique se définit comme un bien collectif, encastré dans des réseaux dans lesquels des briques de connaissances et des capacités d'apprentissage sont combinées afin de générer de nouvelles connaissances. Les flux de connaissances ne sont plus seulement de simples externalités, au sens d'un échange intentionnel, les connaissances circulent également au travers des réseaux d'échange et de partage volontaires (Powell et Grodal, 2005). Dans ce cadre-là, la performance 
innovante ne s'appréhende pas uniquement par le portefeuille de brevets de la firme isolée, mais selon sa position dans des réseaux, lieux de circulation et de production de connaissances.

\section{Charisme géographique vs. invention collective dans les clusters : le rôle de la distance cognitive}

Les clusters sont souvent appréhendés comme des lieux intensifs en connaissances et qui tirent leur attractivité de leur charisme géographique (Appold, 2005). Le capital symbolique que dégage grand nombre de clusters (Dalla Pria et Vicente, 2006), les politiques de marketing territorial qui s'appuient sur ce capital (Longhi, 1999) et les labels « Silicon (.) » ou « (.) Valley »(Vicente et Suire, 2007) constituent souvent des facteurs d'attractivité pour les firmes qui veulent être identifiées comme appartenant à un territoire innovant. Le phénomène peut rapidement devenir autorenforçant, dès lors que les entrepreneurs déduisent que le nombre de firmes innovantes localisées en un lieu est un indicateur de la pertinence du choix de localisation. La politique des pôles de compétitivité s'appuie largement sur cette vision des clusters, en essayant à travers l'identification de quelques pôles à " vocation mondiale » de faire valoir le capital symbolique d'un territoire autour de compétences innovantes particulières.

L'autre vision des clusters sur laquelle s'est également construite la politique des pôles de compétitivité peut-être appréhendée à travers les propriétés de la connaissance cidessus définies. Les clusters apparaitraient alors comme une forme particulière de réseaux d'entreprises innovantes, dans lesquels les firmes « optimisent» l'arbitrage entre appropriation des connaissances et accessibilité aux connaissances sur la base d'une proximité géographique. On peut néanmoins s'interroger sur l'ambivalence des effets de la proximité géographique sur l'innovation. La production de connaissance au sein des réseaux serait-elle conditionnelle à la co-localisation des firmes sur un même espace? Pour saisir cette ambivalence, il faut se placer au cœur du dilemme entre appropriation et accessibilité. Si la propriété intellectuelle fournit théoriquement les moyens à la firme de s'approprier les bénéfices de son activité de création, elle ne protège pas nécessairement la firme de la fuite des compétences et des savoirs développés au cours de l'activité de production de connaissance, avant même que cette dernière soit arrivée à terme. Dans ce cas-là, la proximité géographique est une source forte de flux involontaires de connaissance (Boschma, 2005) qui engendrent des défauts d'appropriation. À l'inverse, la proximité géographique est une source potentielle d'accessibilité à la connaissance dès lors qu'elle facilite les interactions volontaires entre les firmes et réduit les difficultés respectives d'absorption.

Cette ambivalence des effets de la proximité géographique peut s'analyser sous un double angle: celui de la distance cognitive qui sépare les firmes (Nooteboom, 2000), en référence aux distances entre la nature des bases de connaissances de chacune des firmes; et celui de la chaîne de valeur de la connaissance, de sa production à sa diffusion sur le marché (Cooke, 2006; Brossard et Vicente, 2007). Si la proximité géographique favorise l'accessibilité aux connaissances provenant de l'environnement technologique ou sectoriel de la firme, elle 
Figure 1 - Distance cognitive optimale

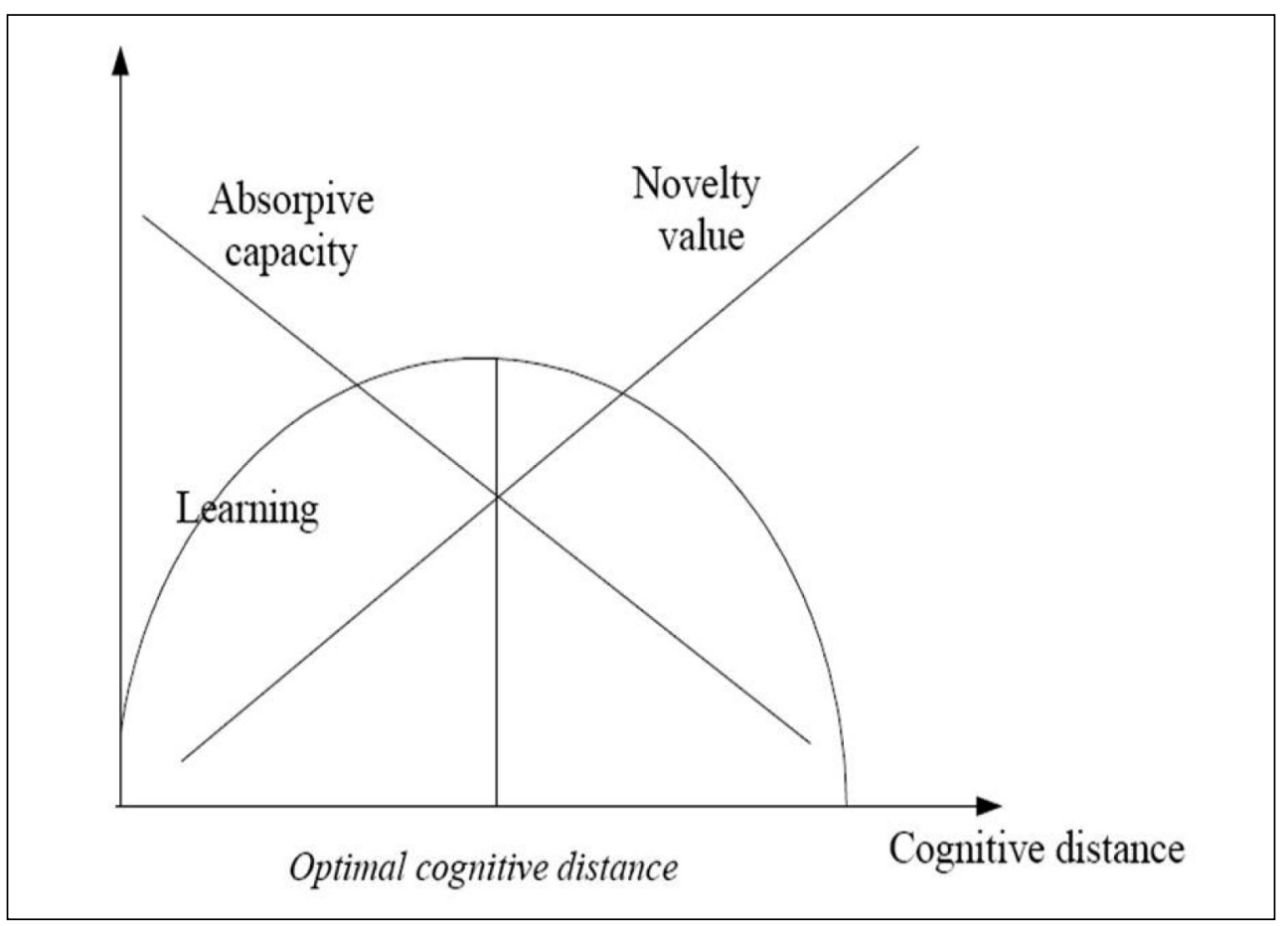

Source: Nooteboom (2000).

présente des risques pour l'appropriation des connaissances produites en interne. Ce risque de défaut d'appropriation sera d'autant plus fort que la distance cognitive qui sépare les firmes est faible (figure 1). En effet, si les firmes présentent des bases de connaissances voisines et opèrent sur des marchés proches, l'absorption de la connaissance de l'une par l'autre sera aisée et les risques de flux de connaissances involontaires - Kogut (1988) et Nooteboom (2000) parlent de hold up - élevés. Une faible distance cognitive fragilise donc la coopération à un niveau local en raison, contrairement à une large part de la littérature, du déficit de confiance que ces risques de défaut d'appropriation peuvent engendrer. À l'inverse, lorsque la distance cognitive atteint un certain seuil, les capacités d'absorption ne sont pas similaires et les risques de flux involontaires plus faibles dès lors que les firmes opèrent sur des marchés suffisamment distincts pour que la coopération entre les deux firmes ne se déroule pas dans un climat de méfiance. La proximité géographique s'accorderait donc mieux d'un certain degré de distance cognitive.

Dans la chaîne de valeur de la connaissance, la distance cognitive est généralement caractéristique des phases exploratoires (Gilsing et Nooteboom, 2006). Généralement, on distingue les phases 
d'exploration des phases d'exploitation de la connaissance. On associe la phase d'exploration à la phase qui consiste à produire une expérimentation et un prototype (beta test) et la phase d'exploitation à la phase de diffusion de la connaissance sur le marché. Or cette typologie doit être remise en cause dès lors que l'on soutient que l'innovation et les stratégies de différenciation des firmes proviennent de la combinaison de connaissances internes avec des connaissances externes, ou dès lors que l'on suppose que la diffusion d'une connaissance sur un marché dépend de son intégration et de sa compatibilité avec un système technique plus large. Cette phase, appelée phase d'« examination » en langue anglaise
(Cooke, 2006; Brossard et Vicente, 2007), est une phase intermédiaire dans laquelle chaque brique de connaissance existe et a été explorée en interne par chacune des firmes, mais nécessite une phase de R\&D complémentaire visant à assurer cette intégration et cette compatibilité dans la perspectives de générer les effets de réseaux auxquels sont très sensibles les consommateurs (Hill, 1997).

Dans les phases exploratoires basées sur des partenariats (tableau 1), un certain degré de distance cognitive est nécessaire, car la connaissance est un bien cumulatif qui émerge de la complémentarité entre des briques de connaissances « distinctes ». Le secteur des TIC est caractéristique d'un tel

Tableau 1 - Flux de connaissances et chaîne de valeur de la connaissance

\begin{tabular}{|c|c|c|c|c|c|}
\hline & & \multicolumn{4}{|c|}{ Caractéristiques de la relation et des flux de connaissances } \\
\hline & & $\begin{array}{l}\text { Degré de } \\
\text { distance } \\
\text { cognitive }\end{array}$ & $\begin{array}{c}\text { Propriété } \\
\text { intellectuelle } \\
\text { et gouvernance }\end{array}$ & $\begin{array}{l}\text { Objectifs } d u \\
\text { partenariat }\end{array}$ & $\begin{array}{c}\text { Degré de } \\
\text { proximité } \\
\text { géographique }\end{array}$ \\
\hline \multirow{3}{*}{ 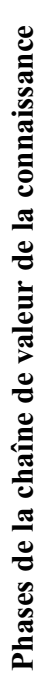 } & Exploration & Fort à moyen & $\begin{array}{l}\text { Cobrevet et } \\
\text { alliance }\end{array}$ & $\begin{array}{l}\text { Développer un beta } \\
\text { test d'une } \\
\text { technologie à partir } \\
\text { de la combinaison } \\
\text { de connaissances } \\
\text { « distantes » }\end{array}$ & Fort \\
\hline & $\begin{array}{c}\text { « Examination » } \\
\text { Phase } \\
\text { d'intégration } \\
\text { et de mise en } \\
\text { compatibilité }\end{array}$ & $\begin{array}{l}\text { Moyen à } \\
\text { faible }\end{array}$ & $\begin{array}{c}\text { Cession de } \\
\text { licence ou } \\
\text { licence croisée } \\
\text { et alliance }\end{array}$ & $\begin{array}{l}\text { Intégrer des modules } \\
\text { de technologies et de } \\
\text { connaissances } \\
\text { existantes }\end{array}$ & $\begin{array}{l}\text { Dépend du degré } \\
\text { de distance } \\
\text { cognitive et des } \\
\text { capacités } \\
\text { respectives } \\
\text { d'absorption }\end{array}$ \\
\hline & Exploitation & Faible & $\begin{array}{l}\text { Création d'une } \\
\text { nouvelle entité, } \\
\text { joint-venture }\end{array}$ & $\begin{array}{c}\text { Capturer des } \\
\text { rendements } \\
\text { d'échelle, diluer le } \\
\text { risque }\end{array}$ & Faible \\
\hline
\end{tabular}


phénomène, car il inclut un grand nombre de technologies génériques (David et Wright, 1999) qui peuvent potentiellement se combiner avec un grand nombre d'autres champs technologiques et sectoriels (tourisme, transport, santé, administrations, espace, médias, etc.). Mais cette phase soulève des problèmes d'apprentissage collectif et d'absorption du fait de la distance cognitive entre les firmes ou entre les ingénieurs et chercheurs de chacune d'elles. Dans ce cadre-là, la fréquence des contacts et la mobilité des ingénieurs et chercheurs favorisent la compréhension mutuelle. La proximité géographique est donc opportune dans les phases exploratoires, et ce d'autant plus que les firmes ne sont pas rivales puisqu'opérant sur des marchés suffisamment distincts. Dans cette phase-là, des partenariats « loose » (peu formalisés et très flexibles) et basés sur la confiance (Gilsing et Nooteboom, 2006) peuvent émerger et être favorisés par la proximité géographique, même si l'output, lui, fait généralement l'objet d'un cobrevet afin d'éviter les conflits de propriété intellectuelle.

Dans les phases dites d'examination, on observe généralement une distance cognitive plus faible mais toujours persistante (Brossard et Vicente, 2007). Les firmes doivent intégrer des modules de connaissance existants afin que chacun puisse tirer partie des externalités de réseaux qui jouent sur la demande des consommateurs (les consommateurs retirent plus d'utilité d'un système complet que des composants du système, ou tirent plus d'utilité de technologies compatibles entre elles). Ainsi, si l'on se repositionne dans le secteur des TIC, les partenariats entre producteurs de technologies connexes portent fréquemment sur ces processus d'intégration (vidéo et téléphonie, navigation et transport, soft et hardware, sécurisation des données et infrastructures, etc.), tout comme les partenariats entre des concurrents, dès lors qu'il s'agit de se positionner sur un standard commun afin de limiter les risques, et assurer une meilleure diffusion de la technologie (Hill, 1997; Shy, 2001). S'il s'agit de producteurs de technologies connexes, un certain degré de distance cognitive demeure et s'accommode alors d'une proximité géographique, même si le fait que les briques de connaissance existent au préalable engendre des partenariats moins « loose » que le cas précédent. Ainsi les accords de licence sont privilégiés pour instaurer a priori un minimum de confiance entre les partenaires, chacun pouvant tirer un avantage du partenariat. Celui qui cède une licence trouve un débouché supplémentaire pour sa technologie en essayant de trouver un partenaire détenteur d'une large " base installée » (Katz et Shapiro, 1994). Celui qui acquiert la licence accroît encore sa base installée en améliorant la qualité et/ou la variété des fonctionnalités proposées par sa technologie. Dans le cadre de partenariats entre concurrents, la proximité géographique est risquée, du fait d'une trop forte proximité cognitive. La proximité temporaire (Rychen et Zimmermann, 2006) entre les ingénieurs et chercheurs dans la définition d'un standard commun permettra alors à chacune des firmes de maintenir un bon niveau d'appropriation tout en favorisant les conditions d'accessibilité.

Enfin, dans la phase collective d'exploitation, la distance cognitive est généralement très faible. Cette phase consiste, une fois le standard établi, à généraliser la connaissance en un « dominant design » (Gilsing et Nooteboom, 2006), afin de tirer partie des 
économies d'échelle et d'assurer la diffusion sur les marchés. Dans ce contexte les firmes peuvent également développer des partenariats où le risque de défaut d'appropriation est fort du fait d'une forte proximité dans les capacités d'absorption respectives des partenaires. La proximité géographique est donc très risquée dans cette phase et les droits de propriété doivent être clairement et formellement spécifiés. Le cas extrême de spécification des droits de propriétés et de gouvernance est la stratégie de joint-venture, stratégie qui consiste alors pour les partenaires à intégrer les activités de production et de marketing dans une unité entièrement dédiée afin de maintenir une distance entre leur activité de production de connaissances.

La production de connaissance est donc un processus collectif qui combine différentes briques de connaissances selon différentes phases de la chaîne de valeur. La proximité géographique, élément-clé de la politique des pôles de compétitivité, devient une caractéristique de la relation entre les détenteurs de briques de connaissance dès lors que l'on se situe en amont de la chaîne de valeur, dans les phases d'exploration et pour partie dans les phases d'examination. La distance cognitive entre les firmes, élément-clé de leur créativité potentielle, caractérise ces phases, et c'est précisément parce que cette distance requiert un accroissement des capacités d'absorption des partenaires respectifs que leur proximité géographique devient un facteur-clé de la créativité.

\section{Les propriétés de stabilité et d'attractivité des clusters}

Propriétés de la connaissance, charisme géographique des clusters et rôle de la distance cognitive dans la production de connaissances constituent donc un ensemble de facteurs qui permettent de discuter des propriétés de performance et de créativité des pôles de compétitivité. Mais pour comprendre comment les clusters peuvent atteindre ce niveau de performance, il faut analyser le processus de colocalisation, et les incitations/motivations qui poussent les firmes à aligner leur choix de localisation sur le choix d'autres firmes.

Appold (2005) a montré que le charisme géographique des clusters peut jouer un rôle fort sur l'attractivité. Ce charisme peut naître d'un processus mimétique selon lequel les firmes décident de leur localisation en fonction de la localisation des firmes qu'elles évaluent comme ayant une certaine réputation dans le secteur dans lequel la firme opère. On retrouve le rôle que peuvent jouer les externalités informationnelles dans les processus de décision: les décisions des prédécesseurs produisent de l'information pour les suiveurs. Si l'on comprend bien la dynamique cumulative que peut engendrer un tel processus - le charisme géographique se renforce au fur et à mesure des localisations successives -, ce charisme ne peut constituer à lui seul une garantie de pérennité et de créativité des clusters, dès lors que ce processus mimétique s'appuie sur une faible distance cognitive entre les firmes. On se situe dans le cadre de ce que Di Maggio et Powell (1983) qualifient d' " isomorphisme mimétique », à savoir l'émergence d'une norme de comportement des organisations suite à la structuration progressive d'un champ organisationnel. Des firmes aux caractéristiques proches font alors l'économie de l'expérimentation de différentes alternatives et alignent leur choix - les choix de localisation 
pour ce qui nous concerne - sur le choix des firmes qu'elles perçoivent comme ayant une forte légitimité dans le champ organisationnel. Un tel isomorphisme se révèle incompatible avec les propriétés du processus collectif de création de connaissances, pouvant même aller jusqu'à en limiter les potentialités.

Dalla Pria et Vicente (2006) ont expliqué et illustré un tel phénomène dans le cas du Silicon Sentier Parisien. Le processus rapide de colocalisation des start-up et dotcom dans le quartier du Sentier - plus de 300 entreprises en moins de 3 années - est né du succès de Yahoo.fr et quelques célèbres suiveurs. Le quartier a très rapidement acquis une forte légitimité, relayée par la presse économique et généraliste, jusqu'à devenir le haut lieu de la net-économie française à la veille de l'éclatement de la bulle internet. Or, la forte proximité cognitive entre les firmes, caractérisée par une forte proximité dans les modèles d'affaires de l'intermédiation, a généré un fort climat de méfiance entre les firmes expliquant la faiblesse des interactions, celle des flux de connaissances, des difficultés dans les capacités d'appropriation, et in fine une faible capacité des firmes à produire de nouvelles connaissances dans un marché qui traversait alors une période de crise. Le Silicon Sentier est rapidement devenu le « Silicon Désert ».

Cet isomorphisme, s'il permet donc de faire émerger le charisme géographique nécessaire à l'identification d'un cluster, n'assure pas pour autant sa capacité à produire durablement de l'innovation. À l'inverse, quand les firmes se colocalisent afin de favoriser leurs conditions d'accessibilité à des connaissances complémentaires, la dynamique change de nature. Les firmes adop- tent un choix de localisation en fonction de l'existence des bases de connaissances présentes localement autour d'un standard technologique, de compétences complémentaires. Le processus est également cumulatif puisque chaque firme vient renforcer le pool de connaissances complémentaires et renforcer ainsi l'attractivité du site. Il est cependant beaucoup moins fragile dès lors que la distance cognitive entre les firmes, tout en accroissant les potentialités d'exploration et d'examination, et donc de créativité, ne vient pas fragiliser les capacités d'appropriation de chacune des firmes.

\section{LE RÔLE DES COMMUNAUTÉS DANS LES RÉSEAUX DE L'INNOVATION}

Si l'on en reste à l'échelle des firmes, c'est donc au niveau de la distance cognitive que se situent les conditions de la performance et de la créativité des pôles de compétitivité. Mais cette créativité n'est que potentielle et requiert des formes de partenariats plus ou moins « loose », plus ou moins codifiées, selon la phase de la chaîne de valeur de la connaissance dans laquelle se situent les firmes. Le dilemme entre appropriation (des) et accessibilité (aux) connaissances qui caractérise chacune des ces phases rend toujours plus ou moins risquée la mise en relation des actifs cognitifs de chacun des partenaires. Pour comprendre comment certains territoires parviennent à activer ce potentiel, il faut sortir du niveau de la firme pour investir le niveau des individus et des dynamiques communautaires (Cohendet et LLerena, 2000; Amin et Cohendet, 2004). C'est au travers de la dynamique de ces communautés, de leurs 
propriétés structurelles, que pourront être alors dessinés les contours d'une approche socio-économique de la performance des pôles de compétitivité

\section{Les clusters comme écosystème : le rôle des réseaux sociaux}

Nous savons qu'il existe des environnements régionaux plus ou moins favorables à l'innovation (Saxenian, 1994; Feldman et Francis, 2002; Lee et al., 2000). La notion d'écosystème se prête assez bien pour décrire des collectifs performants comme la Silicon Valley. Ce territoire regroupe en son cœur des sociétés de hautes technologies, du capital humain qualifié voire créatif (Florida, 2002), des universités et des laboratoires et en sa périphérie, des sociétés de venture capital, des avocats, des chasseurs de têtes, des sociétés de conseils, etc. Et si le territoire apparaît comme une norme mondiale de localisation (Vicente et Suire, 2007) c'est moins lié à la proximité géographique qu'aux relations interfirmes et à la densité des réseaux sociaux qui parcourent la vallée. Cette formidable capacité à se renouveler et à initier des cycles technologiques, à devenir une plateforme créative d'où émergent un certain nombre de standards technologiques fait la différence avec d'autres territoires.

AnnaLee Saxenian les qualifie de " nouveaux argonautes »(Saxenian, 2006). Ils sont ces étrangers de la Silicon Valley, ceux qui tissent des réseaux sur le territoire californien avant de repartir en Chine, en Inde, à Taiwan... Le succès des greffes territoriales qui sont opérées par la suite, s'explique en partie par la connexion qui existe entre les différentes places. En effet, en quittant la vallée, si ces entrepreneurs se déracinent, ils ne renoncent pas pour autant à un actif relationnel accumulé sur place. Précisément, les travaux de Saxenian se concentrent sur les trois groupes les plus importants de la Silicon Valley, à savoir la première génération d'immigrants en provenance de Chine, de Taïwan et d'Inde. Cette première génération a fortement contribué à la réussite de la Silicon Valley et participe activement aux réseaux sociaux et professionnels locaux. Le point important est qu'ils ont maintenu des liens forts dans leurs pays d'origine, maintien rendu possible notamment par les avancées récentes en termes de télécommunications et de transports. Et lorsqu'ils retournent dans leur pays d'origine, ils reproduisent les méthodes de réseaux employées dans la Silicon Valley et maintiennent des liens forts avec des professionnels de la Silicon Valley, ce qui permet alors d'assurer des allers et retours dans la diffusion des connaissances.

En effet, si les connaissances circulent de la Silicon Valley vers des clusters étrangers, elles circulent aussi des clusters étrangers vers la Silicon Valley. Ici c'est donc le maintien de relations à distance et une dynamique entre communautés au sein et hors du territoire qui alimentent la performance collective mais aussi individuelle. En effet, de jeunes entrepreneurs qui souhaiteraient faire appel à des ressources complémentaires et/ou financières trouveront un écho favorable auprès des communautés ethniques déjà constituées sur le territoire. D'une certaine manière, en restant connectés, ils conservent le bénéfice de leur stock de relations sociales mais ils connectent aussi la vallée avec des places distantes qui à leur tour vont se développer sur leur propre sentier de croissance. Ainsi, si l'on se connecte sur la Silicon Valley, l'on 
observe qu'elle est également connectée à un ensemble de territoires innovants et ceci lui confère des propriétés très particulières notamment au regard de sa capacité collective à la créativité. Le succès d'un tel cluster repose donc bien sur la superposition des relations locales et non locales, celles-là même qui permettent aux firmes de se positionner de façon optimale sur l'ensemble des phases de la chaîne de valeur de la connaissance.

\section{Diversité/créativité et communauté : les clusters comme plate-forme relationnelle et technologique}

Plus que l'innovation, la créativité devient désormais une explication à la différence des performances régionales. Cependant son contour reste extrêmement flou (Markusen, 2006) et du point de vue des territoires, l'on mélange souvent l'attraction d'une classe créative - l'agrégation des talents (Florida, 2002) - avec les conditions endogènes de la créativité - la combinatoire des talents -.

Beaucoup a déjà été écrit sur la dimension psychologique et socio-psychologique de la création (Amabile, 1996; Uzzi et Spiro, 2005). Sternberg et Lubart (1999) définissent la créativité comme « l'habileté à produire un travail qui doit être nouveau (original et inattendu) et appropriable (c'est-à-dire utile) ». Boden (1994) suggère pour sa part que la créativité trouve son fondement dans la recombinaison de connaissances existantes et que « ces nouvelles combinaisons doivent être porteuses car pour qualifier une idée de créative, il ne faut pas qu'elle soit simplement nouvelle mais intéressante ». Et cette capacité à former des recombinaisons originales est rarement dévolue à un individu désocialisé. En effet, les psychologues Milliken et al. (2003) expliquent que si les caractéristiques cognitives d'un groupe social sont plutôt homogènes, alors la capacité collective mais aussi individuelle à une recombinaison en rupture est limitée, la diversité cognitive alimentant très largement le potentiel créatif. Dans la lignée des travaux sur le groupthink d'Irving Janis (1972), Suire (2004) montre que la cristallisation d'une position est d'autant plus probable que les individus avec lesquels les interactions sociales sont fréquentes adoptent la même position, ce qui pour Janis s'explique par le fait que « les membres du groupe tendent plus à fournir des efforts pour maintenir l'unanimité que pour rechercher des solutions alternatives »».

À l'inverse, l'échiquier social s'anime à mesure que les réseaux sociaux perdent en cohérence ou en homophilie (Mc Pherson et al., 2001) au profit d'un gain en diversité de position. La confiance et l'acceptation de la différence, étant deux éléments-clés sous-jacents de la créativité. Ainsi le groupe social devient un lieu de créativité se rapprochant du « $\mathrm{Ba}$ » de Nonaka et Konno (1998) à mesure qu'il transcende les frontières cognitives en favorisant la confrontation des idées et des positions.

L'étude de Dibiaggio et Ferrary (2003) sur la dynamique des communautés au sein des clusters de hautes technologies illustre bien ce point. En effet, les auteurs montrent que la performance territoriale au sens d'une capacité collective à innover, est étroitement associée non seulement à la dynamique intercommunautaire mais aussi à la complémentarité des communautés. Ce que l'on définit comme des frontières sociales (Suire, 2006) - le lieu où se nouent les échanges critiques et où s'articulent les 
liens forts et les liens faibles - dessinent les contours d'un creuset de la créativité et façonnent les réseaux de l'entrepreneur.

Rappelons la définition de Granovetter $(1973,2005)$ qui différencie les liens faibles comme ceux que l'on tisse avec l'extérieur d'une communauté d'appartenance ou de pratique et les liens forts comme les interactions typiquement intracommunautaires. D'une certaine manière cette articulation peut se rapprocher de la distinction faite entre interactions locales (liens forts) et interactions distantes (liens faibles) dans les réseaux « petits mondes» (Watts, 1999). Et ces deux formes de réseaux ne semblent aucunement antagonistes bien au contraire, et comme le soulignent Benko et Lipietz (2000), la Silicon Valley peut-être considérée comme un réseau de réseaux, autrement dit, un réseau de " petits mondes » (small worlds), dont les performances en termes de circulation des flux de connaissances se révèlent être en adéquation avec la nécessité pour les firmes de conserver des capacités d'appropriation de leurs propres connaissances tout en maintenant un niveau d'accessibilité aux connaissances complémentaires provenant de leur environnement (Zimmermann, 2001).

La notion de liens forts renvoie aux liens tissés au sein d'un groupe social homogène et cohérent (une famille, les amis, une ethnie, etc.). Ils nécessitent du temps pour s'établir tandis que les liens faibles relèvent de la dynamique intercommunautaire, (les amis de mes amis et plus généralement les connaissances de mes connaissances ou des relations triadiques, etc.) sont moins gourmands en temps et le plus souvent la conséquence de rencontres nouvelles. Cependant, ces liens faibles sont d'une importance considérable puisqu'ils favorisent la capta- tion d'informations en provenance de l'extérieur d'une clique sociale. En un sens, ils favorisent les comportements de rupture ou tout du moins préviennent des risques du conformisme. À l'inverse, les agents qui en sont dépourvus s'inscrivent le plus souvent dans des comportements routiniers et des pratiques très faiblement innovantes. Les communautés de pratiques mais aussi épistémiques ainsi que l'encastrement de certains individus situés à l'intersection d'entre elles, permettent dans une très large mesure d'expliquer le succès d'un territoire fortement innovant comme la Silicon Valley (Dibiaggio et Ferrary, 2003) tout en alimentant des actifs relationnels forts (Storper, 1997). Et c'est là un résultat auquel sont déjà arrivés Cohendet et Llerena (2000) concernant la mise en rapport entre la dynamique d'exploration de connaissances nouvelles et la dynamique intercommunautaire au sein des organisations. Les liens forts sont moins discriminants et ont plus valeur de bien public, au sens où la ressource informationnelle est distribuée plus rapidement sur l'ensemble des agents qui partagent ce communautarisme, en permettant une meilleure exploitation des connaissances sur les marchés. En revanche, ils favorisent les effets de conformisme et de polarisation rapide. Autrement dit, tout le monde se heurte au risque " d'avoir la même idée au même moment ». Avec ces relations intracommunautaires, tout ce qui peut s'écarter trop radicalement de la routine ou de la norme est alors susceptible de rencontrer la désapprobation d'un ou de plusieurs membres du groupe. Ces relations sociales en définitive favorisent très peu les combinaisons originales, les expérimentations nouvelles et la créativité. 
Le travail de Dibiaggio et Ferrary (2003) illustre bien la façon dont fonctionnent ces communautés sur le territoire de la Silicon Valley. Ils ont étudié le fonctionnement de la communauté Française présente au sein de ce cluster. Elle est constituée de 4500 personnes et l'on retrouve ses membres parmi un ensemble très varié d'organisations: universités, société de capital risque, sociétés d'avocats, etc. mais aussi au sein d'institutions non professionnelles comme l'école française de Palo Alto, la maison française, le ciné-club, les restaurants français... Cette communauté trouve à s'exprimer lors de célébrations comme la fête nationale du 14 juillet, la fête du beaujolais nouveau et aussi à travers des journaux... La multiplicité des encastrements permet à chacun d'entretenir un flux de relations sociales qui alimente les relations intracommunautaires qui constituent les liens forts de la communauté française. Ce creuset favorise l'accès à des opportunités et notamment des sources de financement puisque sur « 49 entreprises de hautes technologies crées par des français sans la Silicon Valley, 43 ont été financées par un ou plusieurs des 12 capitaux risqueurs français exerçant leur activité dans la Valley au sein de société de capital risque américaines ou françaises. " (Dibiaggio et Ferrary, 2003). C'est bien l'articulation et la porosité de communautés hétérogènes et complémentaires qui fondent à la fois l'entrepreneuriat et la créativité individuelle par un ensemble de boucles de rétroactions positives successives qui alimentent très largement la performance du territoire. Cohen et Levinthal (1990) ont mis en évidence qu'une organisation est plus que la simple somme de ses talents, ce qui pour Nonaka et al. (1998, 2000) signifie qu'une organisation ne peut créer de nouvelles connaissances en ellemême, elle ne peut le faire sans les individus et leurs interactions. Et de manière similaire, la diffusion interorganisationnelle de connaissances ne peut se faire sans les individus qualifiés et leurs interactions, et ce quel que soit le degré de proximité technologique ou de proximité géographique (Grossetti et Bès, 2004).

Aussi, un territoire qui dégagerait de nombreuses externalités relationnelles en localisant sur son espace des liens forts et/ou des liens faibles, au cœur de la créativité, contribue très rapidement à élever les coûts de sortie. C'est de l'animation de cet espace social que dépend pour partie la performance du collectif. Lee Fleming et Matt Marx (2006) ont cartographié les réseaux d'inventeurs de la Silicon Valley et du territoire de Boston sur la côte Est. Ils montent de façon assez nette que si la Silicon Valley présente des critères de performance, c'est aussi là où le maillage s'approche le plus des caractéristiques de type petits mondes et intercommunautaires. L'accent est particulièrement mis sur le rôle-clé, joué par les individus gatekeepers (Rychen et Zimmeramnn, 2006). Ces « passeurs de connaissances » comblent en effet des trous structuraux (Burt, 2004) et ils sont, comme nous l'avons dit, à l'interface des différentes bases de connaissances en étant les mieux à même de négocier les distances cognitives, celles-là même qui permettent de créer les opportunités d'exploration collective de nouvelles connaissances ou d'examiner la faisabilité des processus d'intégration de connaissances existantes. Mais s'ils sont si cruciaux pour les organisations, ils sont aussi les plus volatils par nature puisque les mieux informés des opportunités. 
Les territoires, parce qu'ils sont le support naturel des interactions sociales, doivent favoriser le maillage des bases de connaissance par l'intermédiaire de ces individus. C'est en stimulant la densité relationnelle et les interactions intercommunautaires que l'on gagne en performance et en compétitivité sur l'ensemble des phases de la chaîne de valeur de la connaissance. Étant entendu que compétitivité s'entend ici comme la propension aux innovations de rupture, celles qui peuvent susciter de nouveaux marchés et in fine, l'établissement de nouveaux standards technologiques, lesquels feront des pôles de compétitivité des lieux certes charismatiques, mais surtout pérennes.

\section{CONCLUSION}

Parce que la connaissance est un bien systémique et complexe, un input et un output de la fonction de production des firmes, la créativité est un processus collectif qui nécessite dans un contexte de convergence technologique d'assembler des briques de connaissances qui proviennent d'environnements parfois distincts. Mais la mise en réseaux de ces actifs cognitifs ne va pas toujours de soi dans un environnement concurrentiel où les firmes doivent toujours maintenir un haut niveau d'appropriation de leurs savoirs, a fortiori quand la proximité géographique peut accroître le risque de flux involontaires de connaissances. Cette proximité géographique pourra néanmoins s'avérer nécessaire dès lors que la distance cognitive entre les firmes, nécessaire à la créativité, ouvre des perspectives d'exploration de nouvelles connaissances, mais requiert de la part des firmes d'ouvrir leurs bases de connaissances et d'avoir une stratégie d'accessibilité réciproque. Face aux risques de propriété intellectuelle que de tels partenariats peuvent faire encourir, l'existence de réseaux sociaux, la formation de communautés inter et intrasectorielles, locales et non locales, sont tout autant de préalables nécessaires à la formation de ces alliances formelles.

Faire reposer le potentiel de l'innovation en France sur la proximité géographique entre les firmes innovantes est donc loin d'être une garantie de la réussite des pôles de compétitivité. Le charisme géographique d'un territoire ne peut être le simple résultat de l'agrégation de talents; c'est dans la recombinaison de ces talents que se situe le potentiel de créativité. C'est dans la capacité des acteurs publics et privés à organiser les réseaux et à imposer les nouvelles connaissances comme des standards technologiques que ce charisme peut devenir pérenne.

\section{BibliograPHIE}

Amabile T., Creativity in Context, Westview Press, 1996.

Amin A., Cohendet P., Architectures of knowledge, Firms, capabilities, and communities, Oxford University Press, New York, 2004.

Antonelli C., "The business governance of localized knowledge: An information economics approach for the economics of knowledge", Industry et Innovation, 13, 2006, p. 227-261. 
Appold S., "The Location Patterns of U.S. Industrial Research: Mimetic Isomorphism, and the Emergence of Geographic Charisma", Regional Studies, vol. 31, n 1, 2005, p. 17-39. Arrow K. J., "Economic welfare and the allocation of resources for invention", The Rate and Direction of Inventive Activity: Economic and Social Factors, Nelson R. R. (ed.), Princeton University Press for N.B.E.R., Princeton, 1962, p. 609-625.

Benko G., Lipietz A., La richesse des régions. La nouvelle géographie socio-économique, PUF, 2000.

Boden M., "Introduction", Dimension of Creativity, Boden M. (eds), MIT Press, 1994.

Boschma R.A., "Proximity and innovation: a critical assessment", Regional Studies 39, 2005, p. 61-74.

Breschi S., Lissoni F., "Knowledge spillovers and local innovation systems: a critical survey”, Industrial and Corporate Change, vol. 10, $\mathrm{n}^{\circ} 4$, 2001, p. 975-1005.

Brossard O., Vicente J., "Cognitive and relational distance in alliances networks: evidence on the knowledge value chain in the European ICT sector", Summer DRUID Conference, Copenhagen, June 2007.

Burt R. S., Structural holes: The social structure of competition, Harvard University Press, Cambridge, MA, 1984.

Cohen W.M., Levinthal D. A., "Absorptive capacity: a new perspective on learning and innovation", Administrative Science Quarterly, 35, 1990, p. 128-152.

Cohendet P., Llerena P., "Routines and the theory of the firm: the role of communities", DRUID Conference, Aalborg, 2000.

Cooke P., Knowledge economics: clusters, learning and cooperative advantage, Routledge, New York, 2002.

Cooke P., "Rational drug design, the knowledge value chain and biosciences megacentres", Cambridge Journal of Economics, 29, 2006, p. 325-341.

Dalla Pria, Y., Vicente J., « Interactions mimétiques et identité collective: gloire et déclin du Silicon Sentier », Revue française de Sociologie, vol. 47, n² 2, 2006, p. 293-317.

David P. A., Wright G., "General purpose technologies and surges in productivity: Historical reflections on the future of the ICT revolution", Stanford Economics working papers, 1999.

Dibiaggio L., Ferrary M., "Communautés de pratiques et réseaux sociaux dans la dynamique de fonctionnement des clusters de hautes technologies", Revue d'économie industrielle, 103, 2003, p. 111-130.

DiMaggio P. J., Powell W. W., “The Iron Gage revisited: Institutional isomorphism and collective rationality in organizational fields", American Sociological Review, vol. 48, $\mathrm{n}^{\circ} 2,1983$, p. 147-160.

Feldman M., Francis J., "Entrepreneurs and the formation of industrial clusters", $3^{e}$ journée de la proximité, Paris, 2002.

Florida R., The rise of the creative class, NY, Basic Books, 2002.

Fleming L., Marx M., "Managing creativity in small worlds", California Management Review, vol. 48, $\mathrm{n}^{\circ}$ 4, 2006, p. 6-27.

Foray D., The economics of knowledge, MIT Press, Cambridge, 2004. 
Gilsing V., Nooteboom B., "Exploration and exploitation in innovation systems: the case of pharmaceutical biotechnology", Research Policy, 35, 2006, p. 1-23.

Granovetter M., "The strength of the weak ties", American Journal of Sociology, 78, 1973, p. $1360-1380$.

Granovetter M., "The impact of social structure on economic outcomes", Journal of Economic Perspectives, 19, 2005, p. 33-50.

Grossetti M., Bès M.P., « Evaluation des effets de proximité: la méthode des histories de relations", 4e journée de la proximité, Marseille, 2004.

Hagerdoorn J., Link A. N., Vonortas N.S., "Research partnerships", Research Policy, 29, 2000, p. 567-586.

Hill C. W., "Establishing a standard: competitive strategy and technological standards in winner-take-all industries", Academy of Management Executive, 11, 1997, p. 7-25.

Iammarino S., McCann P., "The structure and evolution of industrial clusters: Transactions, technology and knowledge spillovers", Research Policy, vol. 35, n 7, 2006, p. 1018-1036,

Katz M., Shapiro C., "Systems competition and network effects", Journal of Economic Perspectives, 8, 1994, p. 93-115.

Kogut B., "Joint Ventures: Theoretical and Empirical Perspectives", Strategic Management Journal, 9, 1988, p. 319-332.

Janis, Irving L., Victims of Groupthink: A Psychological Study of Foreign-Policy Decisions and Fiascoes, Boston, Houghton Mifflin Company, 1972.

Lee C.M., Miller W., Hancock M., Rowen H., Miller W.F (eds), The silicon Valley Edge: Habitat for Innovation and Entrepreneurship, Stanford University Press, 2000.

Longhi C., "Networks, collective learning and technology development in innovative high technology regions: the case of "Sophia-Antipolis", Regional Studies, vol. 33, n 4, 1999, p. 333-342.

Markusen A., "Urban development and the politics of a creative class: evidence from the study of artists", Environment and Planning A, 38, 2006, p. 1921-1940.

Marshall A., Principes d'économie politique, Gordon et Breach, 1890.

McPherson J.M, Smith-Lovin L., Cook J., "Birds of a feather: homophily in social networks", Annual Review of Sociology, 27, 2001, p. 415-444.

Milliken F. J., Bartel C. A., Kurtzberg T. R., "Diversity and creativity in work groups: a dynamic perspective on the affective and cognitive processes that link diversity and performance", Paulus P. B. (eds), Group Creativity, Oxford University Press, 2003.

Mowery D. C., Oxley J.E. And Silverman B. S., "Strategic alliances and interfirm knowledge transfer", Strategic Management Journal, 17, 1996, p. 77-91.

Nonaka I., Konno N., "The concept of 'Ba': building a foundation for knowledge creation", California Management Review, vol. 40, n 3, 1998, p. 40-54.

Nonaka I., Toyoma N., "A firms as a knowledge creating entity: a new perspective on the theory of the firm", Industrial and Corporate Change, 9, 2000, p. 1-20.

Nooteboom B., "Learning by interaction: absorptive capacity, cognitive distance and governance", Journal of Management and Governance, 4, 2000, p. 69-92. 
Powell W.W., Grodal S., "Networks of innovators", The Oxford handbook of innovation, Faberger J. et al., Oxford University Press, New York, 2005, p. 56-85.

Rychen F., Zimmermann J. B., "Clusters in the global knowledge based economy: knowledge gatekeepers and temporary proximity", Proximity Congress, Bordeaux, June 2006.

Saxenian A., Regional Advantage: Culture and Competition in Silicon Valley and Route 128, Harvard, Harvard University Press, 1994.

Saxenian A., The new Argonauts, Harvard University Press, 2006.

Shy O., The economics of network industries. Cambridge University Press, Cambridge, 2001.

Sorenson O., Rivkin J.W., Fleming L., "Complexity, Networks and Knowledge Flow", Research Policy, vol. 35, $\mathrm{n}^{\circ}$ 7, 2006, p. 994-1017.

Sternberg R. J., Lubart T.I., "The concept of creativity: prospects and paradigms”, Handbook of Creativity, Sternberg R.J. (eds), Cambridge University Press, 1999.

Storper M., The regional world: territorial development in a global economy, Guilford Press, 1997.

Suire R., « Des réseaux de l'entrepreneur aux ressorts du creatif: quelles stratégies pour les territoires? », Revue Internationale des PME, vol. 17, 2004, p. 123-143.

Suire R., "Creative cluster and relational proximity", Canadian Journal of Regional Science, 28, 2006, p. 124-138.

Suire R., Vicente, "Why do some places succeed when others decline? A social interaction model of cluster viablility", CREM and LEREPS working paper, 2008.

Ter Wal A., Boschma R., "Co-evolution of firms, industries and networks in space”, Papers in Evolutionary Economic Geography, Utrecht University, 2007.

Uzzi B., Spiro J., "Collaboration and creativity: the small world problem", American Journal of Sociology, 111, 2005, p. 447-504.

Vicente J., Suire R., "Informational cascades vs network externalities in locational choice: evidences on ICT clusters formation and stability", Regional Studies, 41, 2007, p. 173-184.

Watts D., Small Worlds: The Dynamics of Networks Between Order and Randomness, Princeton University Press, 1999.

Zimmermann J.B., "The firm/territory relationships in the globalization: towards a new rationale", European Journal of Economic and Social Systems, 15, 2001, p. 57-76. 